\title{
WEAK COUPLING LIMIT OF THE $N$-PARTICLE SCHRÖDINGER EQUATION*
}

\author{
CLAUDE BARDOS ${ }^{\dagger}$, FRANÇOIS GOLSE ${ }^{\ddagger}$, AND NORBERT J. MAUSER M $^{\S}$
}

\begin{abstract}
This work is devoted to the derivation of a nonlinear 1-particle equation from a linear $N$-particle Schrödinger equation in the time dependent case. It emphazises the role of a so-called "finite Schrödinger hierarchy" and of a limiting (infinite) "Schrödinger hierarchy". Convergence of solutions of the first to solutions of the second is established by using "physically relevant" estimates ( $L^{2}$ and energy conservation) under very general assumptions on the interaction potential, including in particular the Coulomb potential. In the case of bounded potentials, a stability theorem for the infinite Schrödinger hierarchy is proved, based on Spohn's idea of using the trace norm and elementary techniques pertaining to the abstract Cauchy-Kowalewskaya theorem. The core of this program is to prove that if the limiting $N$-particle distribution function is factorized at time $t=0$, it remains factorized for all later times.
\end{abstract}

We offer this contribution to Cathleen Morawetz as an expression of our admiration and friendship and in recognition of the influence that her work on the interaction of mathematics and physics has exerted on us.

1. Introduction: Scalings, hierarchies and formal derivations. The subject matter of this paper is the derivation of a nonlinear 1-particle Schrödinger equation from the linear $N$-particle Schrödinger equation in the so-called weak coupling limit. Both bounded and unbounded potentials, including the case of the Coulomb potential (leading to the Schrödinger-Poisson system) are considered.

The asymptotic relations between the various linear and nonlinear models considered here, starting from the linear $N$-particle Schrödinger equation, are abstracted in the following diagram

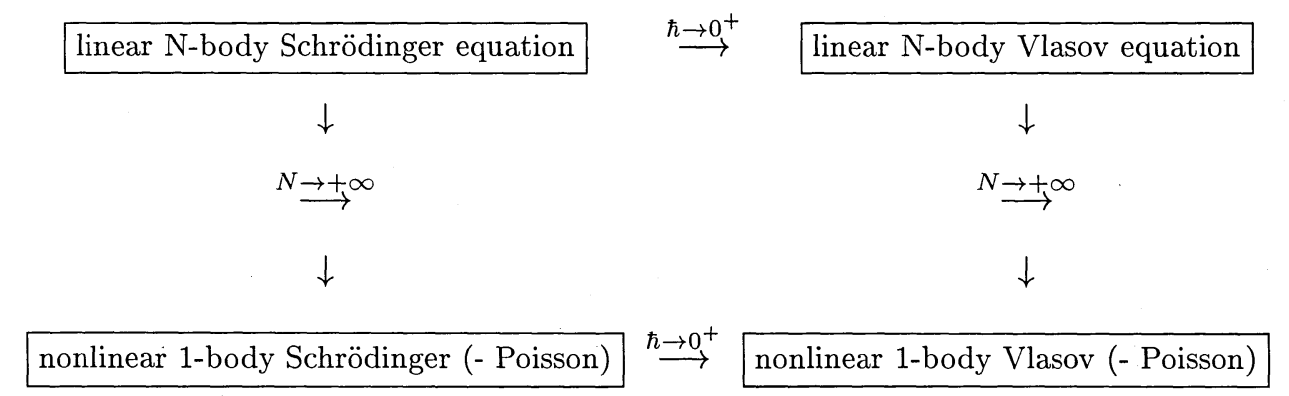

We are concerned with the leftmost vertical arrow, i.e. the "weak coupling" limit and do not discuss the horizontal arrows i.e. the "classical limits" (see [9], [12] for the case of the Schrödinger-Poisson system). Likewise, the diagonal limit, i.e. the " classical + weak coupling limit" corresponding to letting simultaneously $\hbar \rightarrow 0^{+}$and $N \rightarrow+\infty$ is not considered in the present work (we refer to [13] for the case of a bounded, real analytic interaction potential). Hence $\hbar$ is considered as fixed in the sequel.

\footnotetext{
*Received May 26, 2000; Revised Aug 14, 2000.

†CMLA, ENS-Cachan and LAN (Univ. Paris 6), France (bardos@math.jussieu.fr).

$\ddagger$ ENS Ulm and LAN (Univ. Paris 6), France (Francois.Golse@ens.fr).

$\S$ Inst. f. Mathematik, Univ Wien, Strudlhofg. 4, A-1090 Wien, Austria (mauser@cma.univie. ac.at).
} 
The starting point of this derivation is the Schrödinger equation for the wave function $\Psi_{N}=\Psi_{N}\left(x_{1}, x_{2}, \ldots, x_{N}, t\right)$ of $N$ interacting particles, which reads

$$
\begin{array}{r}
i \hbar \partial_{t} \Psi_{N}=-\frac{\hbar^{2}}{2} \sum_{1 \leq j \leq N} \Delta_{x_{j}} \Psi_{N}+\frac{1}{N} \sum_{1 \leq j<k \leq N} V\left(\left|x_{j}-x_{k}\right|\right) \Psi_{N} \\
\Psi_{N}(t=0)=\Psi_{N}^{I}\left(x_{1}, x_{2}, \ldots, x_{N}\right)
\end{array}
$$

The factor $1 / N$ in front of the potential $V$ is the standard "weak coupling scaling", as discussed e.g. by Spohn in [18]. It corresponds to assuming that collective effects of order 1 can be observed over a unit length of the macroscopic time scale.

The potential $V$ is assumed to be real-valued and bounded from below, but no assumption is made as to its sign. In other words, attractive as well as repulsive interactions are amenable to the methods presented in this paper.

The following notations will be used constantly in the sequel

$$
\begin{aligned}
X_{N} & :=\left(x_{1}, x_{2}, \ldots, x_{N}\right), X_{n}:=\left(x_{1}, x_{2}, \ldots, x_{n}\right), X_{N}^{n}:=\left(x_{n+1}, \ldots, x_{N}\right) \\
Y_{N} & :=\left(y_{1}, y_{2}, \ldots, y_{N}\right), Y_{n}:=\left(y_{1}, y_{2}, \ldots, y_{n}\right), Y_{N}^{n}:=\left(y_{n+1}, \ldots, y_{N}\right) \\
Z_{N} & :=\left(z_{1}, z_{2}, \ldots, z_{N}\right), Z_{n}:=\left(z_{1}, z_{2}, \ldots, z_{n}\right), Z_{N}^{n}:=\left(z_{n+1}, \ldots, z_{N}\right)
\end{aligned}
$$

The state of the $N$-particle system can also be described (see for example [8]) by the density operator $\rho_{N}(t)$ acting on $L^{2}\left(\mathbb{R}^{3}\right)^{N}$ or equivalently by its integral kernel, known as the density matrix $\rho_{N}\left(X_{N}, Y_{N}, t\right)$. For a general "mixed state" we have

$$
\rho_{N}\left(X_{N}, Y_{N}, t\right)=\sum_{k \in \mathbb{N}} \lambda_{k} \Psi_{N, k}\left(X_{N}, t\right) \overline{\Psi_{N, k}\left(Y_{N}, t\right)}
$$

where $\lambda_{k}>0$ are the "occupation probabilities" satisfying $\sum_{k} \lambda_{k}=1$. However, the $N$-particle Schrödinger equation is linear, so that we can assume without loss of generality that the density matrix is that of a "pure state":

$$
\rho_{N}\left(X_{N}, Y_{N}, t\right)=\rho_{N}\left(x_{1}, x_{2}, \ldots, x_{N}, y_{1}, y_{2}, \ldots, y_{N}, t\right)=\Psi_{N}\left(X_{N}, t\right) \overline{\Psi_{N}\left(Y_{N}, t\right)}
$$

This density matrix $\rho_{N}\left(X_{N}, Y_{N}, t\right)$ is the integral kernel of the density operator $\rho_{N}(t)$, the time evolution of which is given by

$$
\rho_{N}(t)=e^{-i \frac{t H_{N}}{\hbar}} \rho_{N}(0) e^{i \frac{t H_{N}}{\hbar}}
$$

i.e. the density operator $\rho_{N}(t)$ satisfies the "von Neumann equation" :

$$
i \hbar \partial_{t} \rho_{N}=H_{N} \rho_{N}-\rho_{N} H_{N} .
$$

Equivalently, the density matrix must satisfy

$$
\begin{aligned}
i \hbar \partial_{t} \rho_{N}\left(X_{N}, Y_{N}, t\right)= & -\frac{\hbar^{2}}{2}\left[\Delta_{X_{N}}-\Delta_{Y_{N}}\right] \rho_{N}\left(X_{N}, Y_{N}, t\right) \\
& +\frac{1}{N} \sum_{1 \leq j<k \leq N}\left[V\left(\left|x_{j}-x_{k}\right|\right)-V\left(\left|y_{j}-y_{k}\right|\right)\right] \rho_{N}\left(X_{N}, Y_{N}, t\right)
\end{aligned}
$$

The operator $\rho_{N}$ is of trace class, its trace being given by:

$$
\operatorname{Tr} \rho_{N}(t)=\int \rho_{N}\left(X_{N}, X_{N}, t\right) d X_{N}=\int\left|\Psi_{N}\left(X_{N}, t\right)\right|^{2} d X_{N}=\int\left|\Psi_{N}^{I}\left(X_{N}\right)\right|^{2} d X_{N}=1
$$


after normalization.

The "marginal distributions" or "partial traces" are introduced according to the formula :

$$
\rho_{N, n}(t):=\operatorname{Tr}_{[n+1, N]} \rho_{N}(t)=\int \rho_{N}\left(X_{n}, Z_{N}^{n}, Y_{n}, Z_{N}^{n}, t\right) d Z_{N}^{n}
$$

We further assume that the initial data satisfy the relation

$$
\begin{aligned}
& \rho_{N}\left(x_{1}, x_{2}, \ldots, x_{n}, y_{1}, y_{2}, \ldots, y_{n}, 0\right) \\
= & \rho_{N}\left(x_{\sigma(1)}, x_{\sigma(2)}, \ldots, x_{\sigma(n)}, y_{\sigma(1)}, y_{\sigma(2)}, \ldots, y_{\sigma(n)}, 0\right)
\end{aligned}
$$

for any permutation $\sigma$ of the set $\{1,2,3, \ldots, N\}$. This encodes the fact that we are considering the statistics of undistinguishable particles. This property is preserved by the time evolution of the von Neumann equation, so that (1.10) implies that

$$
\begin{aligned}
& \rho_{N}\left(x_{1}, x_{2}, \ldots, x_{n}, y_{1}, y_{2}, \ldots, y_{n}, t\right) \\
= & \rho_{N}\left(x_{\sigma(1)}, x_{\sigma(2)}, \ldots, x_{\sigma(n)}, y_{\sigma(1)}, y_{\sigma(2)}, \ldots, y_{\sigma(n)}, t\right)
\end{aligned}
$$

holds for all $t \in \mathbb{R}$.

Assuming that the initial $N$-particle distribution satisfies (1.10), we obtain from a rather straightforward computation that the marginal distributions $\rho_{N, n}(t)$ solve the system

$$
\begin{aligned}
& i \hbar \partial_{t} \rho_{N, n}\left(X_{n}, Y_{n}, t\right) \\
= & -\frac{\hbar^{2}}{2}\left[\Delta_{X_{n}}-\Delta_{Y_{n}}\right] \rho_{N, n}\left(X_{n}, Y_{n}, t\right) \\
& +\frac{1}{N} \sum_{1 \leq j<k \leq n}\left[V\left(\left|x_{j}-x_{k}\right|\right)-V\left(\left|y_{j}-y_{k}\right|\right)\right] \rho_{N, n}\left(X_{n}, Y_{n}, t\right) \\
& +\frac{N-n}{N} \sum_{1 \leq j \leq n} \int\left[V\left(\left|x_{j}-z\right|\right)-V\left(\left|y_{j}-z\right|\right)\right] \rho_{N, n+1}\left(X_{n}, z, Y_{n}, z, t\right) d z .
\end{aligned}
$$

Observe indeed that the missing term in (1.12) is the one corresponding to applying the partial trace $\operatorname{Tr}_{[n+1, N]}$ to the summation that appears as the last term in the right hand side of (1.7) restricted to the subset of indices $\{(j, k) \mid n+1 \leq j, k \leq$ $N\}$. Since this restricted sum involves only terms that obviously vanish on the set $\left\{\left(X_{N}, Y_{N}\right) \mid X_{N}^{n}=Y_{N}^{n}\right\}$, applying the partial trace $\operatorname{Tr}_{[n+1, N]}$ does not contribute any additional term in (1.12).

The system (1.12) is called the " $N$-particle (finite) Schrödinger hierarchy". Observe in particular that for $n=N$ one recovers the equation (1.7) for $\rho_{N, N}=\rho_{N}$.

Introducing the operators $\mathcal{C}_{n, n+1}$ mapping $n+1$-particle densities to $n$-particle functions formally defined by

$$
\begin{aligned}
\mathcal{C}_{n, n+1}\left(\rho_{N, n+1}\right)\left(X_{n}, Y_{n}\right):= & \\
& \sum_{1 \leq j \leq n} \int\left[V\left(\left|x_{j}-z\right|\right)-V\left(\left|y_{j}-z\right|\right)\right] \rho_{N, n+1}\left(X_{n}, z, Y_{n}, z\right) d z,
\end{aligned}
$$

the $N$-particle Schrödinger hierarchy is rewritten as:

$$
i \hbar \partial_{t} \rho_{N, n}\left(X_{n}, Y_{N}, t\right)=-\frac{\hbar^{2}}{2}\left[\Delta_{X_{n}}-\Delta_{Y_{n}}\right] \rho_{N, n}\left(X_{n}, Y_{n}, t\right)
$$




$$
\begin{aligned}
& +\frac{1}{N} \sum_{1 \leq j<k \leq n}\left[V\left(\left|x_{j}-x_{k}\right|\right)-V\left(\left|y_{j}-y_{k}\right|\right)\right] \rho_{N, n}\left(X_{n}, Y_{n}, t\right) \\
& +\frac{N-n}{N}\left(\mathcal{C}_{n, n+1} \rho_{N, n+1}\right)\left(X_{n}, Y_{n}, t\right), \quad \forall n=1, \ldots, N, \\
& \rho_{N, n}\left(X_{n}, Y_{n}, t\right)=0, \quad \forall n>N .
\end{aligned}
$$

The "infinite Schrödinger hierarchy" is obtained from the $N$-particle (finite) Schrödinger hierarchy by letting $N \rightarrow+\infty$ while keeping $\hbar$ fixed and giving up the constraint (1.15). We denote by $\rho_{n}$ the $n$-particle marginal distribution involved in the infinite Schrödinger hierarchy which of course differs from $\rho_{N, n}$, the $n$-th marginal distribution involved in the $N$-particle (finite) hierarchy. Letting formally $N \rightarrow+\infty$ in (1.12) leads to:

$$
\begin{aligned}
i \hbar \partial_{t} \rho_{n}\left(X_{n}, Y_{n}, t\right) & =-\frac{\hbar^{2}}{2}\left[\Delta_{X_{n}}-\Delta_{Y_{n}}\right] \rho_{n}\left(X_{n}, Y_{n}, t\right) \\
& +\sum_{1 \leq j \leq n} \int\left[V\left(\left|x_{j}-z\right|\right)-V\left(\left|y_{j}-z\right|\right)\right] \rho_{n+1}\left(X_{n}, z, Y_{n}, z, t\right) d z
\end{aligned}
$$

A function $\rho_{n}$ of the variables $\left(X_{n}, Y_{n}\right)$ is henceforth said to be factorized if it is the $n$-th fold tensor power of a function $\rho \equiv \rho\left(x_{1}, y_{1}\right)$, i.e.

$$
\rho_{n}\left(X_{n}, Y_{n}\right)=\prod_{1 \leq k \leq n} \rho\left(x_{k}, y_{k}\right)
$$

Observe that if $\psi(x, t)$ is a solution of the (nonlinear) "self-consistent, 1-particle Schrödinger equation"

$$
i \hbar \partial_{t} \psi(x, t)=-\frac{\hbar^{2}}{2} \Delta_{x} \psi(x, t)+\int V(|x-z|)|\psi(z, t)|^{2} d z \cdot \psi(x, t)
$$

then

$$
\rho=\psi(x, t) \overline{\psi(y, t)}
$$

is a solution of the "self-consistent von Neumann equation"

$i \hbar \partial_{t} \rho(x, y, t)=-\frac{\hbar^{2}}{2}\left[\Delta_{x}-\Delta_{y}\right] \rho(x, y, t)+\int[V(|x-z|)-V(|y-z|)] \rho(z, z, t) d z \cdot \rho(x, y, t)$,

while the (sequence of) factorized $n$-particle densities

$$
\rho_{n}\left(X_{n}, Y_{n}, t\right)=\prod_{1 \leq k \leq n} \rho\left(x_{k}, y_{k}, t\right)
$$

is a solution of the (infinite) Schrödinger hierarchy. On the other hand, at $t=0$ (cf (1.8)):

$$
\begin{aligned}
\rho_{N, n+1}\left(X_{n}, Y_{n}, 0\right) & =\prod_{1 \leq k \leq n} \psi\left(x_{k}, 0\right) \overline{\psi\left(y_{k}, 0\right)} \prod_{n+1 \leq k \leq N} \int\left|\psi\left(z_{k}\right)\right|^{2} d z_{k} \\
& =\prod_{1 \leq k \leq n} \psi\left(x_{k}, 0\right) \overline{\psi\left(y_{k}, 0\right)}=\prod_{1 \leq k \leq n} \rho\left(x_{k}, y_{k}, 0\right)
\end{aligned}
$$


As a consequence a uniqueness result for the hierarchy (Corollary 5.3 below) implies that, with initial data factorized as in (1.17), the solution of the hierarchy is given by

$$
\rho_{n}\left(x_{n}, y_{n}, t\right)=\prod_{1 \leq k \leq n} \psi\left(x_{k}, t\right) \overline{\psi\left(y_{k}, t\right)}
$$

with $\psi\left(x_{k}, t\right)$ solution of the self-consistent Schrödinger equation (1.18). The factorization, assumed at $t=0$ for the finite hierarchy, will in general get lost at later times due to the presence of the interaction potential $V$; however it is recovered in the limit as $N \rightarrow+\infty$.

In the sequel we establish two types of results:

1) under very general assumptions (containing in particular the physically relevant case of the Coulomb potential) the solution of the finite hierarchy is shown to converge to the solution of the infinite hierarchy;

2) for bounded potentials, the infinite hierarchy is shown to possess a single solution determined by its initial data; in particular, the limit as $N \rightarrow+\infty$ of the sequence $\rho_{N, n+1}$ is factorized and coincides with the functions given by the self-consistent, 1 particle, nonlinear Schrödinger equation.

However, our method for the proof of uniqueness does not encompass the case of the Coulomb potential, and fails to provide a derivation of the Schrödinger-Poisson equation which remains an open problem.

We conclude this introduction by recalling why the Cauchy problem for the $N$ particle Schrödinger equation is well-posed.

THEOREM 1.1. Assume that the real-valued interacting potential $V(|x|)$ is of the form

(1.24) $V(|x|)=V_{1}(|x|)+V_{2}(|x|)$ with $V_{1}(|x|) \in L^{2}\left(\mathbb{R}^{3}\right)$ and $V_{2}(|x|) \in L^{\infty}\left(\mathbb{R}^{3}\right)$.

Then

1. The operator $H_{N}$ defined in $L^{2}\left(\mathbb{R}^{3 N}\right)$ by

$$
H_{N} \Psi=\frac{\hbar^{2}}{2} \sum_{1 \leq j \leq N} \Delta_{x_{j}} \Psi+\frac{1}{N} \sum_{1 \leq j<k \leq N} V\left(\left|x_{j}-x_{k}\right|\right) \Psi
$$

with $D\left(H_{N}\right)=H^{2}\left(\mathbb{R}^{3 N}\right)$ is self-adjoint. In particular, $i H_{N}$ is the generator of a unitary group.

2. for any $\psi^{I} \in H^{1}\left(\mathbb{R}^{3}\right)$, the self-consistent, 1-particle Schrödinger equation (1.18) with initial data $\psi(\cdot, 0)=\psi^{I}$ has a unique solution $\psi \in C\left(\mathbb{R} ; H^{1}\left(\mathbb{R}^{3}\right)\right)$.

The proof of point 1. can be found in Kato's book [6] (Chapter 5, section 5, Remark 5.6), with details in [7]. As for point 2., it is a particular case of Theorem 3.1 in [4] (see also [2]).

2. Preliminaries on trace class operators. As will be shown below the trace norm is the relevant physical quantity used both in the existence proof for the infinite Schrödinger hierarchy with a locally $L^{2}$ potential and in the stability result on this hierarchy in the case of a bounded potential. Indeed, the operators $\rho_{N, n}(t)$ are nonnegative and of trace class with trace norm

$$
\int \rho_{N, n}\left(X_{n}, X_{n}, t\right) d X_{n}=\operatorname{Tr} \rho_{N}(t)=1 .
$$


In order to use this property, the following result concerning trace class operators on products of Hilbert spaces is needed. While it seems elementary, we could not find it proved or even explicitly stated anywhere else. First we recall the following classical notations: let $H_{1}$ and $H_{2}$ denote two Hilbert spaces. The set of HilbertSchmidt operators from $H_{1}$ to $H_{2}$ is denoted by $\mathcal{L}^{2}\left(H_{1}, H_{2}\right)$; the set of bounded operators on $H_{1}$ is denoted by $\mathcal{L}\left(H_{1}\right)$ while $\mathcal{L}^{1}\left(H_{1}\right)$ designates the set of trace class operators on $H_{1}$. We recall that $\mathcal{L}^{1}\left(H_{1}\right)$ is an ideal in $\mathcal{L}\left(H_{1}\right)$, while $\mathcal{L}^{2}\left(H_{1}, H_{2}\right)$ is an $\mathcal{L}\left(H_{1}\right)-\mathcal{L}\left(H_{2}\right)$ bimodule. We refer to [5] $\$ 19.1$ for the basic definitions concerning trace class and Hilbert-Schmidt operators.

LEMMA 2.1. Let $H_{0}:=L^{2}\left(\mathbb{R}^{3 n} \times \mathbb{R}^{3 m}\right)$ and $H_{1}:=L^{2}\left(\mathbb{R}^{3 n}\right)$. Let $K \in \mathcal{L}^{1}\left(H_{0}\right)$ have the integral kernel $k \equiv k\left(X_{n}, Z_{m}, Y_{n}, W_{m}\right)$ :

$$
(K \phi)\left(X_{n}, Z_{m}\right):=\int_{\mathbb{R}^{3 n}} \int_{\mathbb{R}^{3 m}} k\left(X_{n}, Z_{m}, Y_{n}, W_{m}\right) \phi\left(Y_{n}, W_{m}\right) d Y_{n} d W_{m} .
$$

Note that in the sequel we use the abbreviative notation $z=Z_{m}$ and $w=W_{m}$. For a.e. $z \in \mathbb{R}^{3 m}$ and a.e. $w \in \mathbb{R}^{3 m}$, let $K_{z, w}$ be the operator on $L^{2}\left(\mathbb{R}^{3 n}\right)$ with integral kernel $k(\cdot, z, \cdot, w)$ :

$$
\left(K_{z, w} \psi\right)\left(X_{n}\right)=\int_{\mathbb{R}^{3 n}} k\left(X_{n}, z, Y_{n}, w\right) \psi\left(Y_{n}\right) d Y_{n}
$$

Then

1. the map $h \mapsto\left[z \mapsto K_{z, z+h}\right]$, defined a.e. in $h \in \mathbb{R}^{3 m}$, eventually extended to $h \in \mathbb{R}^{3 m}$ as a continous function, belongs to $C\left(\mathbb{R}^{3 m} ; L^{1}\left(\mathbb{R}^{3 m} ; \mathcal{L}^{1}\left(L^{2}\left(\mathbb{R}^{3 n}\right)\right)\right)\right)$,

2. for a.e. $z \in \mathbb{R}^{3 m}, K_{z, z} \in \mathcal{L}^{1}\left(H_{1}\right)$,

3.

$$
\operatorname{Tr}\left|\int_{\mathbb{R}^{3 m}} K_{z, z} d z\right| \leq \int_{\mathbb{R}^{3 m}} \operatorname{Tr}\left|K_{z, z}\right| d z \leq \operatorname{Tr}|K|
$$

Proof. Let $(U,|K|)$ be the polar decomposition of $K$. Hence

$$
K=L_{2}^{*} L_{1} \text { with } L_{1}:=|K|^{1 / 2} \in \mathcal{L}^{2}\left(H_{0}\right) \text { and } L_{2}:=|K|^{1 / 2} U^{*} \in \mathcal{L}^{2}\left(H_{0}\right) .
$$

The operators $L_{1}$ and $L_{2}$ belong to $\mathcal{L}^{2}\left(H_{0}\right)$ and thus have integral kernels $l_{1}$ and $l_{2}$ in $L^{2}\left(\mathbb{R}^{3 n} \times \mathbb{R}^{3 m} \times \mathbb{R}^{3 n} \times \mathbb{R}^{3 m}\right)$, with

$$
k\left(X_{n}, z, Y_{n}, w\right)=\iint \overline{l_{2}\left(X_{n}{ }^{\prime}, Z_{m}{ }^{\prime}, X_{n}, z\right)} l_{1}\left(X_{n}{ }^{\prime}, Z_{m}{ }^{\prime}, Y_{n}, w\right) d X_{n}{ }^{\prime} d Z_{m}{ }^{\prime}
$$

In particular, for a.e. in $z$ and $w \in \mathbb{R}^{3 m}$ :

$$
l_{1}(\cdot, \cdot, \cdot, w) \in L^{2}\left(\mathbb{R}^{3 n} \times \mathbb{R}^{3 m} \times \mathbb{R}^{3 n}\right) \text { and } l_{2}(\cdot, \cdot, \cdot, z) \in L^{2}\left(\mathbb{R}^{3 n} \times \mathbb{R}^{3 m} \times \mathbb{R}^{3 n}\right) .
$$

Hence the operators $L_{1 \mid w}$ with kernel $l_{1}(\cdot, \cdot, \cdot, w)$ and $L_{2 \mid z}$ with kernel $l_{2}(\cdot, \cdot, \cdot, z)$ belong to $\mathcal{L}^{2}\left(H_{1}, H_{0}\right)$ for those values of $z$ and $w$, which is a.e. in $\mathbb{R}^{3 m}$. Again for those values of $z$ and $w, K_{z, w} \in \mathcal{L}^{1}\left(H_{1}\right)$ since, as can be checked directly from (2.4), $K_{z, w}=L_{2 \mid z}^{*} L_{1 \mid w}$. In particular,

$$
\left\|K_{z, z+h}-K_{z, z+h^{\prime}}\right\|_{\mathcal{L}^{1}\left(H_{1}\right)} \leq\left\|L_{2 \mid z}\right\|_{\mathcal{L}^{2}\left(H_{1}, H_{0}\right)}\left\|L_{1 \mid z+h^{\prime}}-L_{1 \mid z+h}\right\|_{\mathcal{L}^{2}\left(H_{1}, H_{0}\right)}
$$


so that, by the Cauchy-Schwarz inequality we have a.e. in $h$ and $h^{\prime} \in \mathbb{R}^{3 m}$

$$
\begin{aligned}
& \int\left\|K_{z, z+h}-K_{z, z+h^{\prime}}\right\|_{\mathcal{L}^{1}\left(H_{1}\right)} d z \\
\leq & \left(\int\left\|L_{2 \mid z}\right\|_{\mathcal{L}^{2}\left(H_{1}, H_{0}\right)}^{2} d z\right)^{1 / 2}\left(\int\left\|L_{1 \mid z+h^{\prime}}-L_{1 \mid z+h}\right\|_{\mathcal{L}^{2}\left(H_{1}, H_{0}\right)}^{2} d z\right)^{1 / 2} \\
= & \left\|L_{2}\right\|_{\mathcal{L}^{2}\left(H_{0}\right)}\left\|l_{1}\left(\cdot, \cdot, \cdot, \cdot+h^{\prime}-h\right)-l_{1}\right\|_{L^{2}\left(\mathbb{R}^{3 n} \times \mathbb{R}^{3 m} \times \mathbb{R}^{3 n} \times \mathbb{R}^{3 m}\right) .}
\end{aligned}
$$

The last factor in the right side of (2.5) converges to zero with $\left|h^{\prime}-h\right|$ since the group of translations acts continuously on $L^{2}$ functions. This establishes point 1 . Point 2 is an obvious consequence of point 1 .

The first inequality in (2.3) being trivial, only the second remains to be proved. Proceeding as in (2.5) shows that

$$
\begin{aligned}
\int\left\|K_{z, z}\right\|_{\mathcal{L}^{1}\left(H_{1}\right)} d z & \leq\left(\int\left\|L_{2 \mid z}\right\|_{\mathcal{L}^{2}\left(H_{1}, H_{0}\right)}^{2} d z\right)^{1 / 2}\left(\int\left\|L_{1 \mid z}\right\|_{\mathcal{L}^{2}\left(H_{1}, H_{0}\right)}^{2} d z\right)^{1 / 2} \\
& =\left\|L_{2}\right\|_{\mathcal{L}^{2}\left(H_{0}\right)}\left\|L_{1}\right\|_{\mathcal{L}^{2}\left(H_{0}\right)}=\left\|\left.K\right|^{1 / 2} U^{*}\right\|_{\mathcal{L}^{2}\left(H_{0}\right)}\left\|\left.K\right|^{1 / 2}\right\|_{\mathcal{L}^{2}\left(H_{0}\right)} \\
& =\|K\|\left\|_{\mathcal{L}^{1}\left(H_{0}\right)}^{1 / 2}\right\| K\left\|_{\mathcal{L}^{1}\left(H_{0}\right)}^{1 / 2}=\right\| K \|_{\mathcal{L}^{1}\left(H_{0}\right)}
\end{aligned}
$$

which implies (2.3).

A direct consequence of relation (2.3) is the following

COROLlary 2.2. The restriction mapping $k \mapsto k(\cdot, z, \cdot, z)$ on functions that are integral kernels of trace class operators is well defined for a.e. $z \in \mathbb{R}^{3 m}$ and induced at the level of integral kernels by $K \mapsto K_{z, z}$ which is a linear contraction mapping from $\mathcal{L}^{1}\left(L^{2}\left(\mathbb{R}^{3 n} \times \mathbb{R}^{3 m}\right)\right)$ with values in $L^{1}\left(\mathbb{R}^{3 m} ; \mathcal{L}^{1}\left(L^{2}\left(\mathbb{R}^{3 n}\right)\right)\right)$.

The linear map $K \mapsto \int K_{z, z} d z$ is continuous from $\mathcal{L}^{1}\left(L^{2}\left(\mathbb{R}^{3 n} \times \mathbb{R}^{3 m}\right)\right)$ to $\mathcal{L}^{1}\left(L^{2}\left(\mathbb{R}^{3 n}\right)\right)$ in the norm topology, but not in the weak-* topology (whose definition is recalled below). Still, the weak-* topology is a natural tool for the convergence of the finite to the infinite Schrödinger hierarchy because of (2.1).

REMARK 2.1. Given a separable Hilbert space $H$, the weak-* topology on $\mathcal{L}^{1}(H)$ is the one induced by the family of semi-norms $T \mapsto|\operatorname{Tr}(K T)|$ indexed by compact operators on $H$. The weak-* topology on $L^{\infty}\left(\mathbb{R}_{+} ; \mathcal{L}^{1}(H)\right)$ is the one defined by the family of semi-norms

$$
T \mapsto\left|\int \operatorname{Tr}(K(t) T(t)) d t\right|
$$

where $K$ runs through $L^{1}\left(\mathbb{R}_{+} ; \mathcal{K}(H)\right)(\mathcal{K}(H)$ denoting the algebra of compact operators on $H)$.

The next lemma provides a crucial argument needed in the course of section 4 .

LEMMA 2.3. Consider a sequence $L_{n} \in \mathcal{L}^{1}\left(L^{2}\left(\mathbb{R}^{d}\right)\right)$ converging to 0 in the weak-* topology, such that the integral kernel $l_{n}$ of $L_{n}$ satisfies

$$
\int\left|l_{n}(z, z+h)-l_{n}(z, z)\right| d z \rightarrow 0 \quad \text { as }|h| \rightarrow 0, \text { uniformly in } n .
$$


Then, for any $\chi \in C_{c}\left(\mathbb{R}^{d}\right)$,

$$
\int l_{n}(z, z) \chi(z) d z \rightarrow 0 \quad \text { as } n \rightarrow+\infty
$$

Proof. Let $\phi \in C\left(\mathbb{R}^{d}\right)$ supported in the unit ball of $\mathbb{R}^{d}$ such that $\phi \geq 0$ and $\int \phi(z) d z=1$. First, because of $(2.7)$

$$
\begin{aligned}
& \left|\int l_{n}(z, z+h) \chi(z) \frac{1}{\epsilon^{d}} \phi\left(\frac{h}{\epsilon}\right) d z d h-\int l_{n}(z, z) \chi(z) d z\right| \\
\leq & \sup _{|h| \leq \epsilon} \int\left|l_{n}(z, z+h)-l_{n}(z, z)\right| \chi(z) d z \int \frac{1}{\epsilon^{d}} \phi\left(\frac{h}{\epsilon}\right) d h \\
\leq & \|\chi\|_{L^{\infty}} \sup _{|h| \leq \epsilon} \int\left|l_{n}(z, z+h)-l_{n}(z, z)\right| d z
\end{aligned}
$$

Hence

(2.8) $\int l_{n}(z, w) \chi(z) \frac{1}{\epsilon^{d}} \phi\left(\frac{w-z}{\epsilon}\right) d z d w \rightarrow \int l_{n}(z, z) \chi(z) d z$, uniformly in $n$ as $\epsilon \rightarrow 0$.

On the other hand, for each $\epsilon>0,(z, w) \mapsto \chi(z) \frac{1}{\epsilon^{d}} \phi\left(\frac{w-z}{\epsilon}\right)$ is the integral kernel of a compact operator on $L^{2}\left(\mathbb{R}^{d}\right)$. Thus, for each $\epsilon>0$,

$$
\int l_{n}(z, w) \chi(z) \frac{1}{\epsilon^{d}} \phi\left(\frac{w-z}{\epsilon}\right) d z d w \rightarrow 0, \text { as } n \rightarrow+\infty \text {. }
$$

The announced result follows from this last statement and the uniform convergence (2.8).

3. A priori estimates for the $N$-particle Schrödinger hierarchy. We start this section with a variant of the Cauchy-Schwarz inequality applied to the marginal distributions. While straightforward, it provides estimates which are useful in the sequel.

Proposition 3.1. The marginal distributions satisfy the inequalities

$$
\iint\left|\rho_{N, n}\left(X_{n}, Y_{n}, t\right)\right|^{2} d X_{n} d Y_{n} \leq 1
$$

and

$$
\left|\rho_{N, n+1}\left(X_{n}, z, Y_{n}, z, t\right)\right| \leq \rho_{N, n+1}\left(X_{n}, z, X_{n}, z, t\right)^{\frac{1}{2}} \rho_{N, n+1}\left(Y_{n}, z, Y_{n}, z, t\right)^{\frac{1}{2}}
$$

for all $t \in \mathbb{R}$.

Proof. The inequality (3.1) follows immediately from the relation:

$$
\begin{aligned}
& \iint\left|\rho_{n}\left(X_{n}, Y_{n}, t\right)\right|^{2} d X_{n} d Y_{n} \\
= & \iint\left|\int \Psi_{N}\left(X_{n}, Z_{N}^{n}, t\right) \overline{\Psi_{N}\left(Y_{n}, Z_{N}^{n}, t\right)} d Z_{N}^{n}\right|^{2} d X_{n} d Y_{n} \\
\leq & \iint\left|\Psi\left(X_{n}, Z_{N}^{n}, t\right)\right|^{2} d X_{n} Z_{N}^{n} \iint\left|\Psi\left(Y_{n}, Z_{N}^{n}, t\right)\right|^{2} d Y_{n} d Z_{N}^{n}
\end{aligned}
$$


while the inequality (3.2) follows from the fact that

$$
\begin{aligned}
& \left|\rho_{N, n+1}\left(X_{n}, z, Y_{n}, z, t\right)\right| \\
= & \left|\int \Psi\left(X_{n}, z, Z_{N}^{n+1}, t\right) \overline{\Psi\left(Y_{n}, z, Z_{N}^{n+1}, t\right)} d Z_{N}^{n+1}\right| \\
\leq & \left(\int\left|\Psi\left(X_{n}, z, Z_{N}^{n+1}, t\right)\right|^{2} d Z_{N}^{n+1}\right)^{1 / 2}\left(\int\left|\Psi\left(Y_{n}, z, Z_{N}^{n+1}, t\right)\right|^{2} d Z_{N}^{n+1}\right)^{1 / 2}
\end{aligned}
$$

Another basic result is a $\hbar$-dependent estimate on the kinetic energy of the $N$ particle system.

Proposition 3.2. Assume that the interacting potential is of the form $V(|x|)=V_{+}(|x|)+V_{-}(|x|)$ with $\left.V_{+}(|x|) \geq 0, V_{+} \in L^{2}\left(\mathbb{R}^{3}\right), V_{-}(|x|)\right) \geq-C_{\text {pot }}>-\infty$.

Assume further that the initial data $\Psi_{N}^{I}\left(x_{1}, \ldots, x_{N}\right)$ satisfies the assumption of indistinguishable particles (1.10) and has the energy

(3.6) $\mathcal{E}_{N, \hbar}$

$$
\begin{aligned}
& =\frac{1}{2} \hbar^{2} \sum_{1 \leq j \leq N} \int\left|\nabla_{x_{j}} \Psi_{N}^{I}\left(X_{N}\right)\right|^{2} d X_{N}+\frac{1}{N} \sum_{1 \leq j<k \leq m} \int V\left(\left|x_{j}-x_{k}\right|\right)\left|\Psi_{N}^{I}\left(X_{N}\right)\right|^{2} d X_{N} \\
& =O(N)
\end{aligned}
$$

as $N \rightarrow+\infty$.

Then, for any $j$ such that $1 \leq j \leq n$, the solution $\Psi_{N}$ of the $N$-particle Schrödinger equation satisfies

$$
\sup _{1 \leq j \leq N} \int\left|\nabla_{x_{j}} \Psi_{N}\left(X_{N}, t\right)\right|^{2} d X_{N} \leq C_{\text {pot }} \frac{N(N-1)}{N^{2} \hbar^{2}}+2 \frac{\mathcal{E}_{N, \hbar}}{N \hbar^{2}} .
$$

Proof. The conservation of energy implies that

$$
\begin{aligned}
& \frac{1}{2} \hbar^{2} \sum_{1 \leq j \leq N} \int\left|\nabla_{x_{j}} \Psi_{N}\left(X_{N}, t\right)\right|^{2} d X_{N}-C_{\mathrm{pot}} \frac{1}{N} \sum_{1 \leq j<k \leq m}\left|\int \Psi_{N}\left(X_{N}, t\right)\right|^{2} d X_{N} \\
\leq & \frac{1}{2} \hbar^{2} \sum_{1 \leq j \leq N} \int\left|\nabla_{x_{j}} \Psi_{N}\left(X_{N}, t\right)\right|^{2} d X_{N}+\frac{1}{N} \sum_{1 \leq j<k \leq m} \int V\left(\left|x_{j}-x_{k}\right|\right)\left|\Psi_{N}\left(X_{N}, t\right)\right|^{2} d X_{N} \\
= & \mathcal{E}_{N, \hbar} .
\end{aligned}
$$

The inequality (3.7) follows from this, (1.8) and the identity

$$
\int\left|\nabla_{x_{j}} \Psi_{N}\left(X_{N}, t\right)\right|^{2} d X_{N}=\frac{1}{N} \sum_{1 \leq j \leq N} \int\left|\nabla_{x_{j}} \Psi_{N}\left(X_{N}, t\right)\right|^{2} d X_{N}, \quad 1 \leq j \leq N,
$$

implied by the assumption of indistinguishable particles.

Corollary 3.3. Under the same assumptions as in Proposition 3.2, and again abbreviating $z=Z_{m}$ and $w=W_{m}$, consider, for any compactly supported continuous function $\Theta$ on $\mathbb{R}^{3 n}$, the sequence of functions $k_{N, n}$ defined by

$$
k_{N, n}(z, w, t)=\iint \Theta\left(X_{n}\right) \Theta\left(Y_{n}\right) \rho_{N, n+1}\left(X_{n}, z, Y_{n}, w, t\right) d X_{n} d Y_{n}
$$


It satisfies the following equicontinuity property:

$$
\sup _{t \in \mathbb{R}} \int\left|k_{N, n}(z, z+h)-k_{N, n}(z, z)\right| d z \leq C\|\Theta\|_{L^{\infty}}^{2}|h|
$$

with

$$
C=\frac{C_{\mathrm{pot}}}{\hbar^{2}}+2 \sup _{N \geq 1} \frac{\mathcal{E}_{N, \hbar}}{N \hbar^{2}}
$$

Proof. In order to avoid cluttered expressions, we deliberately omit the variable $t$, which appears only as a parameter. First

$$
\begin{aligned}
& \int\left|k_{N, n}(z, z+w)-k_{N, n}(z, z)\right| d z \\
= & \int \mid \iiint \Theta\left(X_{n}\right) \Psi_{N}\left(X_{n}, z, Z_{N}^{n+1}\right) \Theta\left(Y_{n}\right) \\
& \times\left[\overline{\Psi_{N}\left(Y_{n}, z+h, Z_{N}^{n+1}\right)}-\overline{\Psi_{N}\left(Y_{n}, z, Z_{N}^{n+1}\right)}\right] d Z_{N}^{n+1} d X_{n} d Y_{n} \mid d z \\
\leq & \|\Theta\|_{L^{2}}^{2}\left\|\Psi_{N}\right\|_{L^{2}}^{2}\left(\iiint\left|\Psi_{N}\left(Y_{n}, z+h, Z_{N}^{n+1}\right)-\Psi_{N}\left(Y_{n}, z, Z_{N}^{n+1}\right)\right|^{2} d Y_{n} d z d Z_{N}^{n+1}\right)^{1 / 2} \\
\leq & \|\Theta\|_{L^{2}}^{2}\left\|\Psi_{N}\right\|_{L^{2}}^{2}\left\|\nabla_{x_{n+1}} \Psi_{N}\right\|_{L^{2}}^{2}|h| .
\end{aligned}
$$

The last inequality is provided by the $L^{2}$ version of the mean value theorem for $H^{1}$ functions:

$$
\int|f(x+h)-f(x)|^{2} d x=\int\left|\int_{0}^{1} \nabla f(x+s h) \cdot h d s\right|^{2} d x \leq\|\nabla f\|_{L^{2}}^{2}|h|^{2} .
$$

One then concludes with the $H^{1}$ estimate (3.7).

4. Convergence of the finite to the infinite Schrödinger hierarchy. The above trace estimates turn out to ensure, with weak assumptions on the interacting potential $V$ and modulo extraction of subsequences, that the marginal distributions $\rho_{N, n}$ converge to a solution of the infinite hierarchy. More precisely:

THEOREM 4.1. Assume that the potential $x \mapsto V(|x|)$ is bounded from below, belongs to $C^{0}\left(\mathbb{R}^{3} \backslash\{0\}\right) \cap L_{\text {loc }}^{2}\left(\mathbb{R}^{3}\right)$ and vanishes at infinity:

$$
\lim _{r \rightarrow+\infty} V(r)=0 \text {. }
$$

Let $\Psi_{N} \in C^{0}\left(\mathbb{R}_{+} ; L^{2}\left(\mathbb{R}^{3}\right)\right)$ be a weak solution of (1.1) with initial data $\Psi_{N}^{I}$ satisfying the assumption of indistinguishable particles (1.10), the normalization (1.8) and with finite energy as in (3.6) where $\mathcal{E}(N, h)=O(N)$ as $N \rightarrow+\infty$. It is assumed that ${ }^{1}$, for all $n \geq 1$,

$$
\rho_{N, n}^{I} \equiv \rho_{N, n}^{I}\left(X_{n}, Y_{n}\right)=\int \Psi_{N}^{I}\left(X_{n}, Z_{N}^{n}\right) \Psi_{N}^{I}\left(Y_{n}, Z_{N}^{n}\right) d Z_{N}^{n} \rightarrow \rho_{n}^{I} \equiv \rho_{n}^{I}\left(X_{n}, Y_{n}\right)
$$

\footnotetext{
${ }^{1}$ Since the problem is linear, the result can trivially be extended to initial data that are finite convex combinations of pure states satisfying the hypothesis.
} 
in $\mathcal{L}^{1}\left(L^{2}\left(\mathbb{R}^{3 n}\right)\right)$ weak-* as $N \rightarrow+\infty$.

Let $\rho_{N}$ be defined by (1.5) with its marginal distributions $\rho_{N, n}$ defined by (1.9). Then, any limit point as $N \rightarrow+\infty$ of the family of partial traces $\left(\rho_{N, n}\right)_{n \geq 1}$ solves the infinite Schrödinger hierarchy (1.16) in the sense of distributions ${ }^{2}$ and satisfies the initial condition

$$
\rho_{n \mid t=0}=\rho_{n}^{I} .
$$

Limit points for the sequence $\left(\rho_{N, n}\right)_{n \geq 1}$ as $N \rightarrow+\infty$ are to be understood in the sense of the product topology on $\Pi_{n \geq 1} L^{\infty}\left(\mathbb{R}_{+} ; \mathcal{L}^{1}\left(L^{2}\left(\mathbb{R}^{3 n}\right)\right)\right)$, each factor being equipped with the weak-* topology.

REMARK 4.1. The assumptions on $V$ in Theorem 4.1 imply those in Theorem 1.1. The assumption on $\mathcal{E}_{N, \hbar}$ implies that Corollary 3.3 applies. In particular, this assumption on $\mathcal{E}_{N, \hbar}$ is satisfied if initial data is factorized:

$$
\Psi_{N}^{I}\left(X_{n}\right)=\prod_{1 \leq j \leq n} \psi\left(x_{j}\right), \quad \int|\psi(x)|^{2} d x=1, \quad \int|\nabla \psi(x)|^{2} d x<+\infty .
$$

REMARK 4.2. The notion of limit points in the product topology described above can be given a somewhat more concrete interpretation by the diagonal extraction procedure. Let $\left(\rho_{n}\right)_{n \geq 1}$ be a weak-* limit point of $\left(\rho_{N, n}\right)_{n \geq 1}$ as $N \rightarrow+\infty$. For each $n \geq 1$, there exists a subsequence of $\rho_{N, n}$ converging to $\rho_{n}$ in $L^{\infty}\left(\mathbb{R}_{+} ; \mathcal{L}^{1}\left(L^{2}\left(\mathbb{R}^{3 n}\right)\right)\right)$ weak-* ${ }^{*}$. In other words, there exists an increasing function $\phi_{n}: \mathbb{N}^{*} \rightarrow \mathbb{N}^{*}$ such that $\rho_{\phi_{n}(N), n} \rightarrow \rho_{n}$ in $L^{\infty}\left(\mathbb{R}_{+} ; \mathcal{L}^{1}\left(L^{2}\left(\mathbb{R}^{3 n}\right)\right)\right)$ weak-*. Define $\phi(N)=\phi_{N} \circ \ldots \circ \phi_{1}(N)$; clearly $\phi(N)$ is an increasing sequence of integers. By the construction of $\phi_{n}$ and the relation (1.15), one sees that $\rho_{\phi(N), n} \rightarrow \rho_{n}$ in $L^{\infty}\left(\mathbb{R}_{+} ; \mathcal{L}^{1}\left(L^{2}\left(\mathbb{R}^{3 n}\right)\right)\right)$ weak-* for all $n \geq 1$. In other words, the same subsequence of $N$ leads to weak-* convergence for all $n \geq 1$.

REMARK 4.3. In the particular case of the Coulomb interaction, the same result holds with the trace norm replaced by the $\mathcal{L}^{2}\left(L^{2}\left(\mathbb{R}^{3 n}\right)\right.$ norm. The proof relies on Leray's $3 D$ variant of the Hardy inequality (formula (1.13) of [10]), called the "uncertainty principle" in [16] and [11].

Proof. As observed in (2.1), the partial trace operators satisfy the estimate

$$
\left\|\rho_{N, n}(t)\right\|_{\mathcal{L}^{1}\left(L^{2}\left(\mathbb{R}^{3 n}\right)\right)}=1, \quad \text { for all } t \in \mathbb{R} .
$$

Thus the sequence $\left(\rho_{N, n}\right)_{n \geq 1}$ indexed by $N$ is relatively compact in $\Pi_{n \geq 1} L^{\infty}\left(\mathbb{R}_{+} ; \mathcal{L}^{1}\left(L^{2}\left(\mathbb{R}^{3 n}\right)\right)\right)$ equipped with its weak-* topology.

Let now $\rho_{\phi(N), n}$ be any weak-* converging subsequence as in Remark 4.2; from now on, we abuse the notation $\rho_{N, n}$ for any such subsequence without further apology.

Since $\mathcal{L}^{1}\left(L^{2}\left(\mathbb{R}^{3 n}\right)\right) \subset \mathcal{L}^{2}\left(L^{2}\left(\mathbb{R}^{3 n}\right)\right)$ with continuous inclusion, the subsequence $\rho_{N, n} \equiv \rho_{N, n}\left(X_{n}, Y_{n}, t\right)$ of marginal distributions converges to $\rho_{n} \equiv \rho_{n}\left(X_{n}, Y_{n}, t\right)$ in $L^{\infty}\left(\mathbb{R}_{+} ; L^{2}\left(\mathbb{R}^{3 n} \times \mathbb{R}^{3 n}\right)\right)$ weak-*, and in particular in $\mathcal{D}^{\prime}\left(\mathbb{R}_{+}^{*} \times \mathbb{R}^{3 n} \times \mathbb{R}^{3 n}\right)$. Thus

\footnotetext{
${ }^{2}$ With the assumptions above, the interaction integrands $\left[V\left(\left|x_{j}-z\right|\right)-V\left(\left|y_{j}-z\right|\right)\right] \rho_{n+1}$ $\left(X_{n}, z, Y_{n}, z, t\right)$ may fail to belong to $L^{1}\left(\mathbb{R}^{3} ; d z\right)$ for each $\left(t, X_{n}, Y_{n}\right)$; yet the integral $\int\left[V\left(\mid x_{j}-\right.\right.$ $\left.z \mid)-V\left(\left|y_{j}-z\right|\right)\right] \rho_{n+1}\left(X_{n}, z, Y_{n}, z, t\right) d z$ is defined as a Radon measure (distribution of order 0 ) in the variables $X_{n}$ and $Y_{n}$.
} 
the free part of the $n$-th equation in the $N$-particle Schrödinger equation converges in the sense of distributions:

$$
i \hbar \partial_{t} \rho_{N, n}+\frac{\hbar^{2}}{2}\left[\Delta_{X_{n}}-\Delta_{Y_{n}}\right] \rho_{N, n} \rightarrow i \hbar \partial_{t} \rho_{n}+\frac{\hbar^{2}}{2}\left[\Delta_{X_{n}}-\Delta_{Y_{n}}\right] \rho_{n}
$$

in $\mathcal{D}^{\prime}\left(\mathbb{R}_{+}^{*} \times \mathbb{R}^{3 n} \times \mathbb{R}^{3 n}\right)$. then

Next consider $\chi \equiv \chi\left(X_{n}, Y_{n}\right)$, a compactly supported continuous test function;

$$
\begin{gathered}
\left|\iint \chi\left(X_{n}, Y_{n}\right)\left[V\left(\left|x_{j}-x_{k}\right|\right)-V\left(\left|y_{j}-y_{k}\right|\right)\right] \rho_{N, n}\left(X_{n}, Y_{n}, t\right) d X_{n}, d Y_{n}\right| \\
\leq\left(\iint\left|\chi\left(X_{n}, Y_{n}\right)\right|^{2}\left(\left|V\left(\left|x_{j}-x_{k}\right|\right)\right|+\left|V\left(\left|y_{j}-y_{k}\right|\right)\right|\right)^{2} d X_{n} d Y_{n}\right)^{\frac{1}{2}} \\
\quad \times\left(\iint\left|\rho_{N, n}\left(X_{n}, Y_{n}, t\right)\right|^{2} d X_{n} d Y_{n}\right)^{\frac{1}{2}} .
\end{gathered}
$$

The first integral in the right hand side of (4.6) is bounded since the potential is assumed locally square integrable; the second integral also is bounded because of the bound on $\left\|\rho_{N, n}(t)\right\|_{\mathcal{L}^{2}}$ implied by Proposition 3.1 and the continuous inclusion $\mathcal{L}^{1}\left(L^{2}\left(\mathbb{R}^{3 n}\right)\right) \subset \mathcal{L}^{2}\left(L^{2}\left(\mathbb{R}^{3 n}\right)\right)$. Thus the second term in the right hand side of (1.12) converges to 0 in $\mathcal{D}^{\prime}\left(\mathbb{R}_{+}^{*} \times \mathbb{R}^{3 n} \times \mathbb{R}^{3 n}\right)$ as $N \rightarrow+\infty$.

The last and most important part of the proof addresses the convergence (in the sense of distributions) of the interaction term, i.e. the third term in the right hand side of (1.12). Consider the three following continuous cut-off functions depending on two arbitrary parameters $0<A<B$ :

$(4.7) 0 \leq U_{A}(r), U_{A B}(r), U_{B}(r) \leq 1, U_{A}(r)+U_{A B}(r)+U_{B}(r)=1$, (4.8) $U_{A}(r)=0$ if $A \geq r, U_{A B}(r)=0$ if $r \leq \frac{A}{2}$, and $B+1 \leq r$, and $U_{B}(r)=0$ if $r<B$.

With the same test function $\chi$ as above, consider the integral

$$
\iiint \chi\left(X_{n}, Y_{n}\right) V\left(\left|x_{j}-z\right|\right) \rho_{N, n+1}\left(X_{n}, z, Y_{n}, z, t\right) d z d X_{n} d Y_{n}=I_{A}^{N}+I_{A B}^{N}+I_{B}^{N}
$$

with the decomposition above defined by

$$
\begin{aligned}
I_{A}^{N} & =\iiint \chi\left(X_{n}, Y_{n}\right) V\left(\left|x_{j}-z\right|\right) \rho_{N, n+1}\left(X_{n}, z, Y_{n}, z, t\right) U_{A}\left(\left|x_{j}-z\right|\right) d z d X_{n} d Y_{n} \\
I_{A B}^{N} & =\iiint \chi\left(X_{n}, Y_{n}\right) V\left(\left|x_{j}-z\right|\right) \rho_{N, n+1}\left(X_{n}, z, Y_{n}, z, t\right) U_{A B}\left(\left|x_{j}-z\right|\right) d z d X_{n} d Y_{n} \\
I_{B}^{N} & =\iiint \dot{\chi}\left(X_{n}, Y_{n}\right) V\left(\left|x_{j}-z\right|\right) \rho_{N, n+1}\left(X_{n}, z, Y_{n}, z, t\right) U_{B}\left(\left|x_{j}-z\right|\right) d z d X_{n} d Y_{n} .
\end{aligned}
$$

We first estimate $I_{A}^{N}$. Without loss of generality, we can assume that $\|\chi\|_{L^{\infty}}=1$ and that the support of $\chi$ is included in $\left(\theta^{-1}(\{1\})\right)^{2 n}$, where $\theta$ is some compactly supported and continuous function on $\mathbb{R}^{3}$. In the sequel, we set

$$
\Theta\left(X_{n}\right)=\prod_{1 \leq k \leq n} \theta\left(x_{k}\right)
$$


By inequality (3.2) in Proposition 3.1,

(4.9) $\left|I_{A}^{N}\right|$

$$
\begin{gathered}
=\left|\iiint \Theta\left(X_{n}\right) \Theta\left(Y_{n}\right) V\left(\left|x_{j}-z\right|\right) \rho_{N, n+1}\left(X_{n}, z, Y_{n}, z, t\right) U_{A}\left(\left|x_{j}-z\right|\right) d z d X_{n} d Y_{n}\right| \\
\leq \iiint\left|\Theta\left(X_{n}\right)\right|\left|\Theta\left(Y_{n}\right)\right|\left|V\left(\left|x_{j}-z\right|\right)\right|\left|\rho_{N, n+1}\left(X_{n}, z, Y_{n}, z, t\right)\right| U_{A}\left(\left|x_{j}-z\right|\right) d z d X_{n} d Y_{n} \\
\leq \iiint\left|\Theta\left(X_{n}\right) \| \Theta\left(Y_{n}\right)\right|\left|V\left(\left|x_{j}-z\right|\right)\right| \rho_{N, n+1}\left(X_{n}, z, X_{n}, z, t\right)^{\frac{1}{2}} \rho_{N, n+1}\left(Y_{n}, z, Y_{n}, z, t\right)^{\frac{1}{2}} \\
\cdot U_{A}\left(\left|x_{j}-z\right|\right) d z d X_{n} d Y_{n}
\end{gathered}
$$

Thus by the Cauchy-Schwarz inequality

$$
\begin{array}{r}
\left|I_{A}^{N}\right|^{2} \leq \iiint\left|\Theta\left(X_{n}\right)\right|^{2}\left|V\left(\left|x_{j}-z\right|\right)\right|^{2} \rho_{N, n+1}\left(Y_{n}, z, Y_{n}, z, t\right) U_{A}\left(\left|x_{j}-z\right|\right) d z d X_{n} d Y_{n} \\
\cdot \iiint\left|\Theta\left(Y_{n}\right)\right|^{2} \rho_{N, n+1}\left(X_{n}, z, X_{n}, z, t\right) U_{A}\left(\left|x_{j}-z\right|\right) d z d X_{n} d Y_{n} .
\end{array}
$$

Taking into account Proposition 3.1 shows that the second integral in the right hand side of $((4.10))$ is bounded by $\|\Theta\|_{L^{\infty}}^{2}$. Integrating first in the $x_{j}$-variable and taking into account (2.3) as well as (2.1) leads to

$$
\begin{aligned}
& \text { (4.11) } \iiint\left|\Theta\left(X_{n}\right)\right|^{2}\left|V\left(\left|x_{j}-z\right|\right)\right|^{2} \rho_{N, n+1}\left(Y_{n}, z, Y_{n}, z, t\right) \mid U_{A}\left(\left|x_{j}-z\right|\right) d z d X_{n} d Y_{n} \\
& \leq\|\theta\|_{L^{1}}^{n-1} \sup _{z \in \mathbb{R}^{3}} \int\left|\theta\left(x_{j}\right)\right|^{2}\left|V\left(\left|x_{j}-z\right|\right)\right|^{2} U_{A}\left(\left|x_{j}-z\right|\right) d x_{j} \iint \rho_{N, n+1}\left(Y_{n}, z, Y_{n}, z, t\right) d Y_{n} d z \\
& =\|\theta\|_{L^{1}}^{n-1} \sup _{z \in \mathbb{R}^{3}} \int\left|\theta\left(x_{j}\right)\right|^{2}\left|V\left(\left|x_{j}-z\right|\right)\right|^{2} U_{A}\left(\left|x_{j}-z\right|\right) d x_{j} .
\end{aligned}
$$

Finally,

$$
\begin{aligned}
\left|I_{A}^{N}\right|^{2} & \leq\|\theta\|_{L^{\infty}}^{2 n}\|\theta\|_{L^{1}}^{n-1} \sup _{z \in \mathbb{R}^{3}} \int\left|\theta\left(x_{j}\right)\right|^{2}\left|V\left(\left|x_{j}-z\right|\right)\right|^{2} U_{A}\left(\left|x_{j}-z\right|\right) d x_{j} \\
& \leq\|\theta\|_{L^{\infty}}^{2 n+1}\|\theta\|_{L^{1}}^{n-1} \int_{|y|<A} V(|y|)^{2} d y .
\end{aligned}
$$

We next estimate $I_{B}^{N}$. Observe that, using again (2.3)

$$
\begin{array}{r}
\left|I_{B}^{N}\right| \leq\|\Theta\|_{L^{2}}^{2} \int\left\|\left(U_{B} V\right)\left(\left|x_{j}-z\right|\right) \rho_{N, n+1}(t)_{z, z}\right\|_{\mathcal{L}^{1}} d z \\
\leq\|\Theta\|_{L^{2}}^{2}\left\|U_{B} V\right\|_{L^{\infty}} \int\left\|\rho_{N, n+1}(t)_{z, z}\right\|_{\mathcal{L}^{1}} d z \\
\leq\|\Theta\|_{L^{2}}^{2}\left\|U_{B} V\right\|_{L^{\infty}}\left\|\rho_{N, n+1}(t)\right\|_{\mathcal{L}^{1}}
\end{array}
$$

Thus, by (2.1)

$$
\left|I_{B}^{N}\right| \leq\|\Theta\|_{L^{2}}^{2}\left\|U_{B} V\right\|_{L^{\infty}}\left\|\rho_{N, n+1}(t)\right\|_{\mathcal{L}^{1}}=\|\Theta\|_{L^{2}}^{2} \sup _{r \geq B}|V(r)| .
$$

Since $V$ is square integrable near $r=0$, the estimate (4.12) shows that

$$
I_{A}^{N} \rightarrow 0 \quad \text { as } A \rightarrow 0^{+} \text {, uniformly in } N \geq 1 \text {. }
$$


Since $V$ tends to 0 as $r \rightarrow+\infty$, the estimate (4.14) shows that

$$
I_{B}^{N} \rightarrow 0 \quad \text { as } B \rightarrow+\infty \text {, uniformly in } N \geq 1 \text {. }
$$

It remains to analyze the term $I_{A B}^{N}$. Without loss of generality, we assume that $\chi$ is supported in $B(0, R)^{2 n}$, where $B(0, R)$ denotes the open ball of radius $R>0$ centered at 0 in $\mathbb{R}^{3}$. Let $\mu \in C_{c}\left(\mathbb{R}^{3}\right)$ such that $\mu \equiv 1$ on $[-B-1-R ; B+1+R]$; obviously

$$
I_{A B}^{N}=\iiint \chi\left(X_{n}, Y_{n}\right) V\left(\left|x_{j}-z\right|\right) U_{A B}\left(\left|x_{j}-z\right|\right) \rho_{N, n+1}\left(X_{n}, z, Y_{n}, z, t\right) \mu(z) d z d X_{n} d Y_{n}
$$

Define then

$$
\begin{aligned}
& l_{N}(z, w, t) \\
= & \iint \Theta\left(X_{n}\right) \Theta\left(Y_{n}\right) V\left(\left|x_{j}-z\right|\right) U_{A B}\left(\left|x_{j}-z\right|\right) \\
& \cdot\left[\rho_{N, n+1}\left(X_{n}, z, Y_{n}, w, t\right)-\rho_{n+1}\left(X_{n}, z, Y_{n}, w, t\right)\right] d X_{n} d Y_{n} .
\end{aligned}
$$

By construction, $l_{N}(\cdot, \cdot, t)$ is the integral kernel of a trace class operator $L_{N}(t)$, satisfying $L_{N} \in L^{\infty}\left(\mathcal{L}^{1}\left(L^{2}\left(\mathbb{R}^{3}\right)\right)\right.$ as well as $L_{N} \rightarrow 0$ in $L^{\infty}\left(\mathcal{L}^{1}\left(\mathbb{R}_{t} ; L^{2}\left(\mathbb{R}^{3}\right)\right)\right.$ weak-* as $N \rightarrow+\infty$. Further, because of Corollary 3.3 and the assumed continuity of $x \mapsto V(|x|)$ on $\mathbb{R}^{3} \backslash\{0\}$, the family $l_{N}$ satisfies the following equicontinuity property:

$$
\int\left|l_{N}(z, z+h, t)-l_{N}(z, z, t)\right| d z \rightarrow 0 \text { uniformly in } N \text { and } t \geq 0 \text { as }|h| \rightarrow 0 .
$$

By Lemma 2.3,

$$
\int l_{N}(z, z, t) \chi(z) d z \rightarrow 0 \text { in } L^{\infty}\left(\mathbb{R}_{+}\right) \text {weak-* as } N \rightarrow+\infty .
$$

Thus

$$
I_{A B}^{N} \rightarrow \iiint \chi\left(X_{n}, Y_{n}\right) V\left(\left|x_{j}-z\right|\right) U_{A B}\left(\left|x_{j}-z\right|\right) \rho_{n+1}\left(X_{n}, z, Y_{n}, z, t\right) d z d X_{n} d Y_{n} .
$$

Grouping (4.15), (4.16) and (4.17) shows that

(4.18) $\mathcal{C}_{n, n+1} \rho_{N, n+1} \rightarrow \mathcal{C}_{n, n+1} \rho_{n+1}$ as $N \rightarrow+\infty$ in $\mathcal{D}^{\prime}\left(\mathbb{R}_{+}^{*} \times \mathbb{R}^{3 n} \times \mathbb{R}^{3 n}\right)$ weak-*.

This establishes the convergence of the finite to the infinite Schrödinger hierarchy.

Finally, (4.5), (4.6) and (4.18) show that, for all $\zeta \in C_{c}^{\infty}\left(\mathbb{R}^{3 n} \times \mathbb{R}^{3 n}\right)$

$$
\partial_{t} \iint \rho_{N, n}\left(X_{n}, Y_{n}, t\right) \zeta\left(X_{n}, Y_{n}\right) d X_{n} d Y_{n} \in L^{\infty}\left(\mathbb{R}_{+}\right)
$$

This establishes the initial condition (4.2).

5. Stability of the Infinite Hierarchy and Factorization. In this section, we make the additional assumption that $V \in L^{\infty}\left(\mathbb{R}_{+}\right)$. The infinite hierarchy can be recast in the abstract form

$$
\hbar \partial_{t} \rho_{n}=A_{n} \rho_{n}+\mathcal{C}_{n, n+1} \rho_{n+1}, \quad n \geq 1
$$


with $\mathcal{C}_{n, n+1}$ defined in (1.13) and $A_{n}$ denoting the skew-adjoint operator

$$
A_{n}=i \frac{\hbar^{2}}{2}\left(\Delta_{X_{n}}-\Delta_{Y_{n}}\right)
$$

The trace norm is a "good" norm for both the operators $A_{n}$ and $\mathcal{C}_{n, n+1}$; this, together with its analogy with the $L^{1}$ norm on functions, makes it a natural tool in studying these Schrödinger hierarchies, as noticed by Spohn [17]. More precisely

\section{Proposition 5.1.}

1. The operator $A_{n}$ is the generator of a unitary group in $\mathcal{L}^{1}\left(L^{2}\left(\mathbb{R}^{3 n}\right)\right)$ ).

2. The operators $\mathcal{C}_{n, n+1}$ are continuous from $\mathcal{L}^{1}\left(L^{2}\left(\mathbb{R}^{3(n+1)}\right)\right)$ ) to $\left.\mathcal{L}^{1}\left(L^{2}\left(\mathbb{R}^{3 n}\right)\right)\right)$

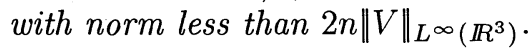

Proof. Statement 1. follows from the definition of the trace norm, the formula

$$
e^{t A_{n}}\left(f_{n}\right)=e^{-i \frac{\hbar^{2}}{2} t \Delta_{n}} \circ f_{n} \circ e^{+i \frac{\hbar^{2}}{2} t \Delta_{n}}
$$

and the fact that $\exp \left(+i \frac{\hbar^{2}}{2} t \Delta_{n}\right)$ is unitary on $L^{2}\left(\mathbb{R}^{3 n}\right)$.

Let $F_{n+1} \in \mathcal{L}^{1}\left(L^{2}\left(\mathbb{R}^{3 n} \times \mathbb{R}^{3}\right)\right)$ with integral kernel $f_{n+1}$, and let $G_{n+1}$ be the operator with integral kernel

$$
g_{n+1}\left(X_{n}, z, Y_{n}, w\right)=V\left(\left|x_{j}-z\right|\right) f_{n+1}\left(X_{n}, z, Y_{n}, w\right)-f_{n+1}\left(X_{n}, z, Y_{n}, w\right) V\left(\left|y_{j}-w\right|\right) .
$$

Since $V \in L^{\infty}, G_{n+1}$ also belongs to $\mathcal{L}^{1}\left(L^{2}\left(\mathbb{R}^{3 n} \times \mathbb{R}^{3}\right)\right)$ and

$$
\left\|G_{n+1}\right\|_{\mathcal{L}^{1}\left(L^{2}\left(\mathbb{R}^{3 n} \times \mathbb{R}^{3}\right)\right)} \leq 2\|V\|_{L^{\infty}}\left\|G_{n+1}\right\|_{\mathcal{L}^{1}\left(L^{2}\left(\mathbb{R}^{3 n} \times \mathbb{R}^{3}\right)\right)} .
$$

Since $\mathcal{C}_{n, n+1} F_{n+1}$ is a sum of $n$ terms of the form

$$
\int\left(G_{n+1}\right)_{z, z} d z
$$

statement 2. follows from Lemma 2.1.

The proposition above naturally leads to the following statement concerning the stability of the infinite hierarchy.

THEOREM 5.2. Assume that $V \in L^{\infty}\left(\mathbb{R}_{+}\right)$, and denote by $H_{n}$ the Banach space $\mathcal{L}^{1}\left(L^{2}\left(\mathbb{R}^{3 n}\right)\right)$. Let $\left(\rho_{n}\right)_{n \geq 1} \in \prod_{n \geq 1} L^{\infty}\left(\mathbb{R}_{+} ; H_{n}\right)$ be a weak solution of the infinite Schrödinger hierarchy (5.1) with initial data $\left(\rho_{n}^{I}\right)_{n \geq 1}$ satisfying

$$
\left\|\rho_{n}^{I}\right\|_{H_{n}} \leq \epsilon_{n}, \quad n \geq 1
$$

where $\left(\epsilon_{n}\right)_{n \geq 1}$ is a given sequence of positive numbers. In addition, assume the existence of a positive constant $D$ such that

$$
\sup _{t \geq 0}\left\|\rho_{n}(t)\right\|_{H_{n}} \leq D, \quad \text { for all } n \geq 1
$$

Then, for all $0<t<\frac{\hbar}{2\|V\|_{L} \infty}$,

$$
\left\|\rho_{n}(t)\right\|_{H_{n}} \leq \sum_{m \geq 0}\left(\begin{array}{c}
n+m \\
m
\end{array}\right)\left(\frac{2\|V\|_{L^{\infty}} t}{\hbar}\right)^{m} \epsilon_{n+m} .
$$


Proof. Set $z_{n}(t)=e^{-t A_{n} / \hbar} \rho_{n}(t)$; the $n$-th equation in the infinite Schrödinger hierarchy (5.1) becomes

$$
\partial_{t} z_{n}(t)=\frac{1}{\hbar} e^{\frac{t}{\hbar} A_{n}} \mathcal{C}_{n, n+1} e^{-\frac{t}{\hbar} A_{n+1}} z_{n+1}(t)
$$

This last relation and Proposition 5.1 imply that, for all $n \in \mathbb{N}^{*}$ and all $t \geq 0$,

$$
\left\|z_{n}(t)\right\|_{H_{n}} \leq \epsilon_{n}+\frac{2\|V\|_{L^{\infty}}}{\hbar} n \int_{0}^{t}\left\|z_{n+1}(s)\right\|_{H_{n+1}} d s
$$

Iterating this inequality leads to

$$
\begin{aligned}
& \left\|z_{n}(t)\right\|_{H_{n}} \leq \epsilon_{n}+\sum_{k=0}^{m}\left(\frac{2\|V\|_{L^{\infty}}}{\hbar}\right)^{k} n(n+1) \ldots(n+k-1) \int_{S_{k}(t)} \epsilon_{n+k} d s_{1} \ldots d s_{k} \\
& +\left(\frac{2\|V\| L_{L^{\infty}}}{\hbar}\right)^{m} n(n+1) \ldots(n+m-1) \int_{S_{m}(t)}\left\|z_{n+m}\left(s_{m}\right)\right\|_{H_{n+m}} d s_{1} \ldots d s_{m}
\end{aligned}
$$

where $S_{k}(t)$ is the $k$-simplex of size $t$, i.e.

$$
S_{k}(t)=\left\{\left(s_{1}, \ldots, s_{k}\right) \mid 0 \leq s_{1} \leq \ldots \leq s_{k} \leq t\right\}
$$

The $k$-dimensional volume of $S_{k}(t)$ is $t^{k} / k$ ! and thus

$$
\left\|z_{n}(t)\right\|_{H_{n}} \leq \sum_{k=0}^{m} \epsilon_{n+k}\left(\begin{array}{c}
n+k \\
k
\end{array}\right)\left(\frac{2\|V\|_{L^{\infty}} t}{\hbar}\right)^{k}+\left(\begin{array}{c}
n+m \\
m
\end{array}\right) D\left(\frac{2\|V\|_{L^{\infty}} t}{\hbar}\right)^{m} .
$$

The last term is equivalent to

$$
m^{n}\left(\frac{2\|V\|_{L^{\infty}} t}{\hbar}\right)^{m} D
$$

for each fixed $n \geq 1$ as $m \rightarrow+\infty$. Thus it vanishes in the limit as $m \rightarrow+\infty$ for all $0<t<\frac{\hbar}{2\|V\|_{L^{\infty}}}$, leading to the announced inequality.

This stability statement obviously implies a uniqueness result for the infinite Schrödinger hierarchy.

COROLlaRY 5.3. Let $\left(\rho_{n}^{1}\right)_{n \geq 1}$ and $\left(\rho_{n}^{2}\right)_{n \geq 1}$ be two solutions of the infinite Schrödinger hierarchy, obtained by the limiting procedure of the previous section and which coincide at time $t=0$. Then they are equal for all $t \in \mathbb{R}_{+}$.

Proof. Indeed, (1.8) implies that

$$
\left\|\rho_{n}^{1}(t)-\rho_{n}^{2}(t)\right\|_{H_{n}} \leq 2, \quad \text { for all } t \geq 0 \text { and } n \in \mathbb{N}^{*} .
$$

Applying Theorem 5.2 to $\left(\rho_{n}^{1}-\rho_{n}^{2}\right)_{n \geq 1}$ with $\epsilon_{n}=0$ for all $n \geq 1$ and $D=2$ proves that

$$
\rho_{n}^{1}(t)=\rho_{n}^{2}(t), \quad n \in \mathbb{N}^{*}, \quad|t|<\frac{\hbar}{2\|V\|_{L^{\infty}}} .
$$

Since the infinite Schrödinger hierarchy is autonomous and the bound (1.8) uniform in time, this implies that $\rho_{n}^{1}(t)=\rho_{n}^{2}(t)$ for all $t \in \mathbb{R}$ and all $n \in \mathbb{N}^{*}$. 
This uniqueness result implies in turn the following factorization property, established by Spohn [17] by a somewhat different method.

THEOREM 5.4. Assume that $V \in L^{\infty}\left(\mathbb{R}_{+}\right)$and that $V(r) \rightarrow 0$ as $r \rightarrow+\infty$. For any $\psi^{I} \in H^{1}\left(\mathbb{R}^{3}\right)$ and $\left\|\psi^{I}\right\|_{L^{2}}=1$, define

$$
\rho_{n}^{I}\left(X_{n}, Y_{n}\right)=\prod_{1 \leq j \leq n} \psi^{I}\left(x_{j}\right) \overline{\psi^{I}\left(y_{j}\right)} .
$$

Let $\rho_{N, n}$ be the weak solution of the finite Schrödinger hierarchy defined by

$$
\rho_{N, n}\left(X_{n}, Y_{n}, t\right)=e^{-i \frac{t}{\hbar} H_{N}} \rho_{n}^{I}\left(X_{n}, Y_{n}\right) e^{+i \frac{t}{\hbar} H_{N}},
$$

where $H_{N}$ is the $N$-body Schrödinger operator defined in (1.25).

Then : As $N \rightarrow+\infty, \rho_{N, n} \rightarrow \rho_{n}$ in $L^{\infty}\left(\mathbb{R}_{+} ; \mathcal{L}^{1}\left(L^{2}\left(\mathbb{R}^{3 n}\right)\right)\right)$ weak-*, with $\rho_{n}$ given by the formula

$$
\rho_{n}\left(X_{n}, Y_{n}, t\right)=\prod_{1 \leq j \leq n} \psi\left(x_{j}, t\right) \overline{\psi\left(y_{j}, t\right)}
$$

where $\psi$ is the solution of the self-consistent, 1-particle Schrödinger equation

$$
i \hbar \partial_{t} \psi(x, t)=-\frac{\hbar^{2}}{2} \Delta \psi(x, t)+\int V(|x-y|)|\psi(y, t)|^{2} d y \psi(x, t)
$$

with initial data

$$
\psi(x, 0)=\psi^{I}(x)
$$

This is a straightforward consequence of Corollary 5.3, since the formula (5.4) defines a solution of the infinite Schrödinger hierarchy which satisfies

$$
\left\|\rho_{n}(t)\right\|_{H_{n}}=\|\psi(t)\|_{L^{2}}^{2 n}=1
$$

because the self-consistent Schrödinger equation leaves the total mass $\|\psi(t)\|_{L^{2}}$ invariant. We refer to [4] for a survey of existing results on the self-consistent SchrödingerPoisson equation.

6. Conclusion. We give a careful presentation and "marginal" improvements of the theory of weak coupling limits that represent the next step towards the derivation of the Schrödinger-Poisson equation.

In 1977 Braun and Hepp [1] derived the Vlasov equation from a system of $N$ classical particles. They assumed the interacting potential bounded in $C^{2}$ and prove the convergence of the classical Liouville hierarchy to the Vlasov hierarchy. They avoided addressing the question of the uniqueness of solutions of the infinite hierarchy, but nevertheless succeeded to prove that the limits of $N$-particle marginals (in the large $N$ limit) coincide with factorized products of solution of the Vlasov equation. Their argument rely on a clever use of probabilistic methods (such as the strong law of large numbers to establish the propagation of chaos and the central limit theorem to analyze fluctuations about the mean field).

Such an argument does not seem to work for the limit $\hbar \rightarrow 0, N \rightarrow+\infty$ of a system of quantum particles. This might explain why Narnhofer and Sewell [13] had to prove a uniqueness theorem for the Vlasov equation. Since the corresponding 
operator is very singular, stringent assumptions (equivalent to the analyticity of the potential) are required in that paper.

Spohn [17] came up with the idea of using the trace norm to prove the convergence of the solution of the $N$-body Schrödinger equation to the factorized $N$-body state driven by the self-consistent Schrödinger equation. His proof rests on the assumption of a bounded interaction potential, as in the previous section of the present paper. Being based on the Duhamel formula, his proof avoids the question of uniqueness for the inifinite Schrödinger hierarchy, although in a way very different from that in [1].

In the present contribution we tried to illustrate the difference between the proof of the uniqueness (with stringent assumptions) and the proof of the convergence of the $N$-body Schrödinger hierarchy to the infinite Schrödinger hierarchy (under fairly general assumptions).

One should observe that the proof of the Theorem 4.1 seems closely related to the proof of the "abstract" Cauchy-Kowalewskaya Theorem according to Nirenberg [14] and Nishida [15]. To make this connection clear one should introduce the scale of spaces:

$$
\mathcal{H}_{\beta}=\left\{\left(\rho_{n}\right)_{n \geq 1} \in \prod_{n \geq 1} H_{n} \text { such that } \sum_{n \geq 1} \beta^{n}\left\|\rho_{n}\right\|_{H_{n}}<+\infty\right\} .
$$

On the other hand the proof of convergence to the infinite hierarchy may be interesting by itself. It is obtained here under very general assumptions and only the physically relevant estimates are involved in the proof.

Indeed, in many cases such an infinite hierarchy appears as a linearization of a nonlinear problem. Therefore the existence of a solution for such an infinite hierarchy may be considered as the existence of some type of very weak solution of the underlying nonlinear problem.

Furthermore, a closer consideration of the proof shows that the assumption of a factorized solution plays no role in the proof of convergence. At this level the only important thing is the assumption of indistinguishable particles. Therefore as observed by Spohn [18] and recalled by Cercignani ([3], p. 264), the solution of the infinite hierarchy also describes systems of a large number of correlated particles, when the $N$-body density matrix is not of the factorized form. It seems the only available description of such systems, where the $N$-body density fails to be factorized (i.e. satisfy what is called the molecular chaos assumption in classical physics, and the Hartree ansatz in the quantum case). Note that the Schrödinger hierarchy itself is the same for the "Hartree-Fock" and for the "Hartree" ansatz. In follow up work together with A. Gottlieb we shall be concerned also with the case of antisymmetrized initial data.

Acknowledgement. This research was supported by the European Union TMR network "Asymptotic methods in kinetic equations" (ERB FMRX CT97 0157) and by the Austrian START project "Nonlinear Schrödinger and quantum Boltzmann equations" of N.J.M. Also, F.G. was supported by the Institut Universitaire de France. The first two authors thank the ESI in Vienna for its hospitality.

We express our gratitude to H.T. Yau for fruitful discussions and to A. Gottlieb for helpful criticisms of the manuscript. 


\section{REFERENCES}

[1] W. BRAUN AND K. HePP, The Vlasov dynamics and its fluctuation in the $1 / N$ limit of interacting particles, Comm. Math. Phys., 56 (1977), pp. 101-113.

[2] T. Cazenave And A. Haraux, Introduction aux Problèmes D'évolution Semi-linéaires,Ellipses, Paris, 1990.

[3] C. Cercignani, Ludwig Boltzmann, Oxford Univ. Press, 1998.

[4] J. Ginibre AND G. Velo, On a class of nonlinear Schrödinger equations with nonlocal interaction, Math. Zeitschr., 169 (1980), pp. 109-145.

[5] L. Hörmander, The Analysis of Linear Partial Differential Operators, vol. 1, Springer Verlag, Berlin, New York, 1983.

[6] T. Kato, Perturbation Theory for Linear Operators, Springer-Verlag, 1966.

[7] T. Kato, Fundamental properties of Hamiltonian operators of Schrödinger type, Trans. Amer. Math. Soc., 70 (1951), pp. 195-211.

[8] L. Landau And E. Lifshitz, Quantum Mechanics, Course in Theoretical Physics 3, Pergamon Press, Oxford, New York, Toronto, 1974.

[9] P. L. Lions And T. PAUl, Sur les mesures de Wigner, Revista Math. Iberoamericana, 9 (1993), pp. $553-617$.

[10] J. LERAY, Sur le mouvement d'un liquide visqueux emplissant l'espace, Acta Math., 63 (1934), pp. 193-248.

[11] E. LIEB AND H.-T. YAU, The Chandrasekhar theory of Stellar collapse as the limit of quantum mechanic, Commun. Math. Phys., 112 (1987), pp. 147-174.

[12] P. A. MARKOWICH AND N. J. MAUSER, The classical limit of a self-consistent quantum-Vlasov equation in 3-D, Math. Mod. and Meth. in Appl. Sciences, 9 (1993), pp. 109-124.

[13] H. NARnhofer AND G. L. SEwell, Vlasov hydrodynamics of a quantum mechanical model, Commun. Math. Phys., 79 (1981), pp. 9-24.

[14] L. Nirenberg, An abstract form of the nonlinear Cauchy-Kowalewski theorem, J. of Diff. Geom., 6 (1972), pp. 561-576.

[15] T. NishidA, A note on a theorem of Nirenberg, J. of Diff. Geom., 12 (1978), pp. 629-633.

[16] M. Reed And B. Simon, Methods of Modern Mathematical Physics, vol. 2, 4th ed., Academic Press, 1987.

[17] H. Spoнn, Kinetic equations from Hamiltonian dynamics, Rev. Mod. Phys., 52:3 (1980), pp. 600-640.

[18] H. Spohn, Quantum kinetic equations, in On the Three Levels, M. Fannes, et al., eds., Plenum Press, New York, 1994, pp. 1-10.

[19] H. Spohn, Boltzmann hierarchy and Boltzmann equation, in Kinetic Theories and the Boltzmann Equation, C. Cercignani, ed., LNM 1048, Springer-Verlag, Berlin, 1984.

[20] W. Thirring, A Course in Mathematical Physics, vol. 4, Springer-Verlag, 1983. 
